NOTICE

Available from:

National Technical Information Service

U.S. Department of Commerce

5285 Port Royal Road

Springfield, Virginia 22161

703-487-4650

Use the following price codes when ordering:

Price: Printed Copy $\mathrm{AO} 3$

Microfiche A01 


\title{
Charge states of high $Z$ atoms in a strong laser field
}

\author{
S.M. Susskind, E.J. Valeo, C.R. Obernan \\ Princeton Plasma Physics Laboratory \\ P.O. Box 451. Princeton, New Jersey 08543 \\ J.B Bernstein \\ Section of Applied Physics, Yale Lniversity \\ Hew Haven, Connecticut 06520
}

\begin{abstract}
We present a numerical solution of the Thomas-Fermi atom in the presence of a static electric field as a model of the adiabatic response of a heavy atom in the presence of a strong laser field. In this semiclassical approach, we calculate the resulting charge state of the atom and its induced dipole moment after the field is turned on. Due to the scaling properties of the Thomas-Fermi ; pproach, the resulting - ztal atomic charge and dipole moment can be expressed as a universal function of the field. We compare our results with recent jonization experiments performed on noble gases using laser fields.
\end{abstract}

\section{DISCLAIMER}

\begin{abstract}
This report was prepared as an account of work sponsored by an agency of the United States Govermment. Neitber the Uni:ed States Government nor any agency thereor, nor any of their employeses. makes any warranty, express or implied, or assumes any legal liability of responsibility for the accuracy. completeness, or usefulness of any information, apparatus. praduct, or process disclosed. or represin's that its use would not infringe privately owned rights Reference herein to any specific comnereial product, process, or service by trade name. Irademark, manufiscluser. or otherwise does not necessarily constilute ur unply its endorsement, recommendation, ar fatoring by the Uaited States Gaternment or any agency thereof The views and opinions of authors expressed herein do nol necessarily state or refiect those of the United States Government or any agency thereot.
\end{abstract}




\section{Introduction}

Madern laser systems can deliver electric field sirengths on the order of or bigger than the electric field exerted by the atomic nucleus on the outer shell electrons. An important question is how do these fields act on matter? A number of ionization experiments have been performed, mostly on noble gases, to answer this question. ${ }^{3,2}$ It is important to notice that in these experiments two different time scales exist: the laser period is much smaller than a characteristic atomic time $h_{h} I_{p}$, where $I_{p}$ is the ionization potential. Therefore, the outer electrons see the laser field as quasi-static and the number of photons absorbed by the atom in the ionization process is large. A second feature of these experiments is the apparent absence of shell effects. In ref. 2. the authors note that their ionization rates cipend very little on the details of the structure of the particular atom under study, but depend only on its binding energy:

Any attempt at explaining these ionization experiments has to be non-perturbative in claracter due to the high applied fields. The disparity in time scales and apparent lack of shell effects have led to attempts to fit the data ${ }^{2}$ with single electron models suth as Jieldysh's. which describes multiphoton ionization from the ground state of a hydrogenic atom to the continuum ignoring the effats of intermediate states.

We will take here the complementaiy approach; we will study the case of a many electron atom where the electron density $\tan$ be calculated semiclassically, and where the screening effect of the inner electrons is taken into account. A semiclassical calculation may be expected to be good for a high $\mathcal{Z}$ atom becaise most of its electrons are in energ: levels with high quantum numbers.

It is clear that any attempt at a calculation at the level of .Hartree-Fock of a system such as a Xenon atom in the presence of a strong field is numerically prohibitive. However, the apparent absence of shell effects and the large number of electrons involved in our problem suggest that a statistical approach such as the Thomas-Fermi model is in order. In this model $_{+}^{3}$ the electronic cloud is considered as a Fermi gas at zero tempera "ure; that is, as a gas of electrons whose energy distribution is a step function at the maxi num possible eriergy (Fermi energy). The electron density is then calculated by filling the available phase space volume using the Pauli exclusion principle. This electron density is then used in Poisson's equation to compute the self-consistent eleccrostatic potential of the atom. The Thomas-Farmi model can also be derived more rigorously from the electron density calculated from the solution of the Hartree-Fock equation if we neglect exchange effects and treat the single-particle wave functions semiclassically. ${ }^{4}$ For a large $Z$ atom, exchange and quantum corrections are both of order $Z^{-2 / 3}$ compared to those retained in the Thomas-Fermi model.

The disparity between the laser frequency and a characteristic atomic frequency sug. gests a straightforward modification of the model to solve the problem of the atom in the presence of the low frequency laser field viz.: The potential due to a constant electric field, corresponding to the peak value of the laser field, is added to the Thomas-Fermi equation. since it is at this time in the laser field period that most of the ionization occurs. 
In the next section, we will describe our model and set up the Thomas-Fermi equation for our system. We present there our numerical results and the universal curves which express the charge state and induced dipole moment of a heavy atom as a function of the applied ficld strengti. The comparison with axailable experimental data is done in section 3 . We conclude with a discussion in section 4 .

\section{Thomas-Fermi atom in an external electric field}

Here, we derive the Thomas-Fermi equation for an atom distorted by an electric field. Our derivation proceeds along the lines of the usual one for the 'bare' Thomas-Fermi aton. Vie present it here mostly for the sake of completeness.

Our sy'stem consists of $Q_{e l}$ electrons around a nucleus of charge $Z$, subject to an external potential $V_{\text {ext }}=-F z$, where the $z$-axis is chosen along the direction of the field. (Atomic units are used throughout.) If we are at a position $r$ and the maximum arailable energy of a single electron in the atom is $-\epsilon$, then, classically, the momentum of the fastest electron at $\mathbf{r}$ is

$$
p_{f}=\sqrt{2[\phi(\mathbf{r})+F z-\epsilon]},
$$

where $-c$ is the energy at the separatrix and $-\phi$ is the effective potential seen by the electron (see Fig.1).

The number of phase space cells at $\mathbf{r}$ is $d^{3} p d V /(2 \pi)^{3}$. Each cell can hold two electrons (one for each spin state) according to Pauli's principle, and if we integrate this number between 0 and $p_{f}(r)$, we have for the electron number density ${ }^{6}$

$$
\eta(r)=\frac{p^{3}}{3 \pi^{2}}=\frac{2^{3 / 2}}{3 \pi^{2}}(\varphi+F z-\epsilon)^{3 / 2} .
$$

The electron density is zero outside the region delimited by the separatrix (outside the classical region). We can use now this constitutive relation between $\phi$ and $n$ together with Poisson s equation $\Gamma^{2} \phi=4 \pi n$ to find the total potential $\phi$;

$$
F^{2} \phi= \begin{cases}8 \sqrt{2} / 3 \pi(\dot{\phi}+F z-\epsilon)^{3 / 2} & \text { if } r<r \\ 0 & \text { otherwise. }\end{cases}
$$

$r_{g}$ is the separatrix curve; the curve that solves the equation $\phi+F z-\epsilon=0$. As $r-0$, the nucleus is not screened, so $\phi-Z / r$ in this limit. The other boundary condition is that as $r \rightarrow \infty . \phi-Q / r$, where $Q$ is the total charge of the atom, $Q \equiv Z-Q_{\mathrm{ei}}$, with

$$
Q_{e i} \equiv \int d V n(\mathbf{r}) \text {. }
$$

Equation (3) is two dimensional, due to the azimuthal symmetry around the field axis.

It is convenient ta change the dependent variable $\phi$ to $x$, where $\phi+F==(Z / r) x$, and the independent rariable $r$ to the Thomas-Fermi scaled coordinates $r=6 Z^{-1 / 3} x$ 
$\left.b \equiv 2^{-\tau / 3}(3 \pi)^{2 / 3}\right)$. With these changes. Eq. (3). Written now in sprerical coordinates $: \mu \equiv \cos 0 \%$, transiorms to

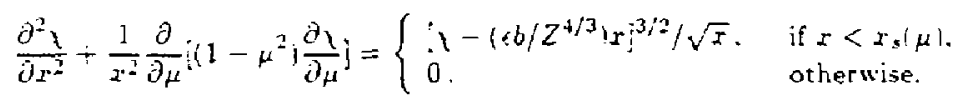

The boundary conditions are now

$$
\left\{\begin{array}{l}
\{(0 . \mu)=1 \\
\left(-Q / Z+\left(F / Z^{5 / 3}\right) b^{2} x^{2} \mu, \text { for } x-x .\right.
\end{array}\right.
$$

aild $Q_{e l}$ takes on the form

$$
\frac{Q_{e l}}{Z}=\frac{1}{2} \int_{-1}^{1} d \mu \int_{0}^{x, \mid \mu)} d x x^{1 / 2}\left(x-\frac{c b}{Z^{4 / 3}} x\right)^{3 / 2} .
$$

We can also obtain an expression for the itpole moment. $D_{2}$, induced by the external field $F$, In analogy with Eq. (i).

$$
D_{z}=<z>\equiv \frac{\int d l n(\mathbf{r}) z}{\int d V n(\mathbf{r})}=\frac{Z^{2 / 3}}{Q_{e l}} \frac{b}{2} \int_{-1}^{1} d \mu \mu \int_{0}^{x_{*}(\mu)} d x x^{3 / 2}\left(x-\frac{e b}{Z^{4 / 3}} x\right)^{3 / 2} .
$$

In $\mathrm{Eq} .(5)$, with boundary conditions given by Eq. (6). $\epsilon$ appears only in the combination $\epsilon Z^{-4 / 3}$ and the field strength $F$ appears only in the combination $F Z^{-5 / 3}$. It is clear then that $6 Z^{-4 / 3}=g\left(F Z^{-5 / 3}\right)$, where $g$ is a function which is independent of $Z$; that is, universal for all (heavy) atoms. In the same way, from Eq. ( $\bar{i}$ ), $Q_{e l} / Z$, and hence $Q / Z=I-Q_{e l} / Z$, are universal functions of $c Z^{-4 / 3}$, and hence of $F Z^{-5 / 3}$, j.e., $Q / Z=h\left(F Z^{-5 / 3}\right)$. The same reasoning holds for the induced dipole moment $D_{z} Z^{1 / 3}$ given by $\mathrm{Eq} .(8)$. It is, therefore, sufficient to calculate the charge states, induced dipole noment, and $\epsilon$ curves rersus applied field strength for $Z=1$ only, which produces the universal curves for all atoms.

We choose to solve $\mathrm{Eq}_{\mathrm{q}}(5)$ for $\chi(x, \mu)$ numerically by a relaxation method. We put an extra $\partial_{x} / \partial t$ in $\mathrm{Eq} .(5)$ and obtain then diffusion-type equation whose solution will converge to the solution of Eq. (j) as $t \rightarrow \infty$. To solve the two-dimensional partial difierential equation, we use an alternating-direction-implicit scheme. ${ }^{7}$ We take as initial conditions for $x$, the well-known Thomas-Fermi function $x T F$ for the bare atom $(F=0)$. (This function satisfies $x_{T}^{\prime \prime} F=x_{T F}^{3 / 2} / x^{1 / 2}$ ). He take $\epsilon=0$ initially. At each subsequent time step we find $\varepsilon$ as follows: Because of the azimuthal symmetry, we know that the saddle point of $\varphi($ hence $x / x)$ is at a point $z=z$, along the $z$ axis. We find this point numerjcally by solving $\partial(x / x) / \partial z=0$. Having $z$, we can calculate the energy at the separatrix as

$$
\epsilon=\frac{Z^{4 / 3}}{b} \frac{x(z s+1)}{z s} \text {. }
$$

Once we know $c$, we can generate the rest of the separatrix curve $x \equiv x s(\mu)$ by solving $x(x, \mu)-\left(\epsilon b Z^{-4 / 3}\right) x=0$ numerically for $x=x_{s}(\mu)$. Kinowing $x_{s}(\mu)$, we can construct the 
updated electron density in the righthand side of Eq. 15 ) and solve again for 1 . repeating the whole process until we achieve self-consistency.

The physical meaning of 6 is, as mentioned before. the maximum energy of a single botend electron in the presence of the external field. The amount rif energy required to extract ar electror. from an ion $x^{+7}$ for $F=0$ is the ionization potential. $I_{F}$. of that charge state $\mathrm{I}^{+7}$. It proves to be more convenient for comparison with experiments to plor the field required to produce a certain charge state versus the ionization potential of that charge state. rather than versus $c$. For that, we need to calculate both the jonization potential of the Thomas-Fermi atom or ion of a certain charge and also the field requited to produce that charge. Again. the result can be expressed in a universal curse for all atoms.

The universal curves for thi total charge of the ion. $Q / Z$, and dipole moment, $D=Z^{1 / 3}$. vetsus field. $F Z^{-5 / 3}$. and intensity. $I Z^{-10 / 3}$. versus ionization potential. $J_{p} Z^{-4 / 3}$. are presented in Figs. 2, 3, and 4 . respectively.

\section{Comparison with Ionization Experiments}

In ref. 2, the authors summarized the results of their experiments in a plot of the threshold intensity for the appearance of a charge state versus the ionization potential of the prerious charge state. They defined the threshold intensity as the intensity required to have a probability of $10^{-4}$ for the appearance of the given charge state. The plot contains the results for Ar, Kr, and Xe, and is reproduced in Fig. 5a.

In our semiclassical model, we cannot calculate the field which yieids $10^{-4}$ probability of ionization, but we can and do calculate the field for an ionization probability equal to 1. This provides an upper bound for the saturation intensity measured in experiments. If we replot Fig. 4 for $Z=18,36$, and 54 and display the results in experimental units, we obtain Fig. 5b. Although we cannot compare Figs. 5a and $5 b$ vis a vis, due to the different definitions of the intensities, it is clear that the model gives the right scaling with the atcmic number $Z$ and that it provides a reasanably close upper bound for the saturation intensity of a given charge state. If we read from the plotted data in ref. 2 the intensities required to have a $100 \%$ ionization yield, we come much closer to the numbers we obtained in our calculations.

It is important to mention the two groups that made the experiment (see refs. 1,2) have differences in their reported intensities for the appeasance of a given ionization species, which in some cases are of two orders of magnitude. The two experiments differ, however. an order of magnitude in their pulse lengths. Our model possibly explains the 'prompt' ejected electrons. which: probably account for most of the ionization signal in a short-pulse, high-intensity experiment. One can expect, however, that the ionization signal in a longpulse experiment will have a contribution from eltctrons which evertually tunnel ihrough the confining potential barrier and which cannot be accounted for by the present model. 


\section{Conclusions}

A useful upper bound for the intensity required to reach a specified charge state by nonresonance ionization of high $Z$ gases has been obtained by employing a siatistical model of the aton subject to a constant electrsc fied. This model takes into account only the bulk and screening properties of the atom and overlooks the details of the atomic structure. In this way, as is seen experimentally, the ionization properties are only a function of the ionization potential. Moreover, the model predicts the right scaling or ordering for the different atoms considered experimentally:

\section{Acknowledgments}

We would like to thank Dr. M.D. Perry for permission to reproduce Fig.3 of ref. 2. We would also like to thank Dr. S.C. Cowley and Prof. R. Kulsrud for useful comments and discussions, and Prof. S. Suckewer for his interest and support. This work was supported by DOE under contracts Xos. KC.05-01 and DE-ACO2-76-CHO3073 and by ONR under Grant $500014-8 i-k \cdot 2006$. 


\section{References}

A. L'Huillier, L.A. Lompré. G. Mainfray and C. Manus. J. Phỵs. B, 16, 1363 (1983); Phys. Rev. A. 27. 2503 (1983).

2M.D. Perry, A. Szöke. O.L. Landen. and E.M. Campbell, Phys. Rev. Lett.. 60, 1270 (1989); M.D. Perry: O.L. Landen. A. Szöke, and E.M. Campbell. Phys. Rev. A. 37. iti (1988).

${ }^{3}$ For a complete description of the Thomas-Fermi approach, together with applications (up to 1948), see P. Gombás, Die Statistische Theorie des Atoms und ihre Anwendungen (Springer, Viena 1949). For an updated review, see X.H. March in Theory of the Inhomogeneous Electron Gas. ed. by S. Lundquist and N.H. March (Plenum, New lork, 1983) ch.1.

${ }^{4}$ D.A. Irirzhnitz, Yu. A. Lozovik, and G.V. Shpatakorskaya, Usp. Fiz. Xauk.. 117. 3 (1975) [Sov. Phys. L'sp., 18, 649 (1976)].

3A.S. Kompaneets and E.S. Pavlovskii. Zh. Eksp. Teor. Fiz, 31, 42 i (1956) [Sor. Phys. JETP, 4, $323(1957)]$.

'This quantization argument. as 'cells' in phase space, does not require the system to be separable. See L.D. Landau and E.M. Lifschitz, Quantum Mechanics (Pergammon, X.Y. 1977) p. 174 .

'R.D. Richtmeyer, and K.W. Morton. Difference Methods for Initial-Value Problems (Interscience, N.Y. 1967), p. 211. 


\section{Figures}

FIG. 1. Sketch of the self-consistent potential. $-o$; external potential $V_{e r t}=-F z$ : and separatrix energy $-c$. The hatched area is where the electron densily is different from zero.

FIG. 2. Universal curve of the total ionic charge, $Q / Z$, versus field $F Z^{-5 / 3}$.

FIG. 3. Tniversal curve of the induced dipole moment, $D_{z} Z^{1 / 3}$, versus field $F Z^{-5 / 3}$.

FIG. 4. Universal curve of the time-averaged intensity, $I Z^{-10 / 3}$, versus ionization potential $I_{p} Z^{-4 / 3}$.

FIG. 5. Intensity versus ionization potential for Xe, Fir, and Ar. (a) Experimental curve of threshold intensitjes required for $10^{-4}$ probability of ionization. (b) Theoretical curve of intensities required for $100 \%$ ionization. 
\#89T0121

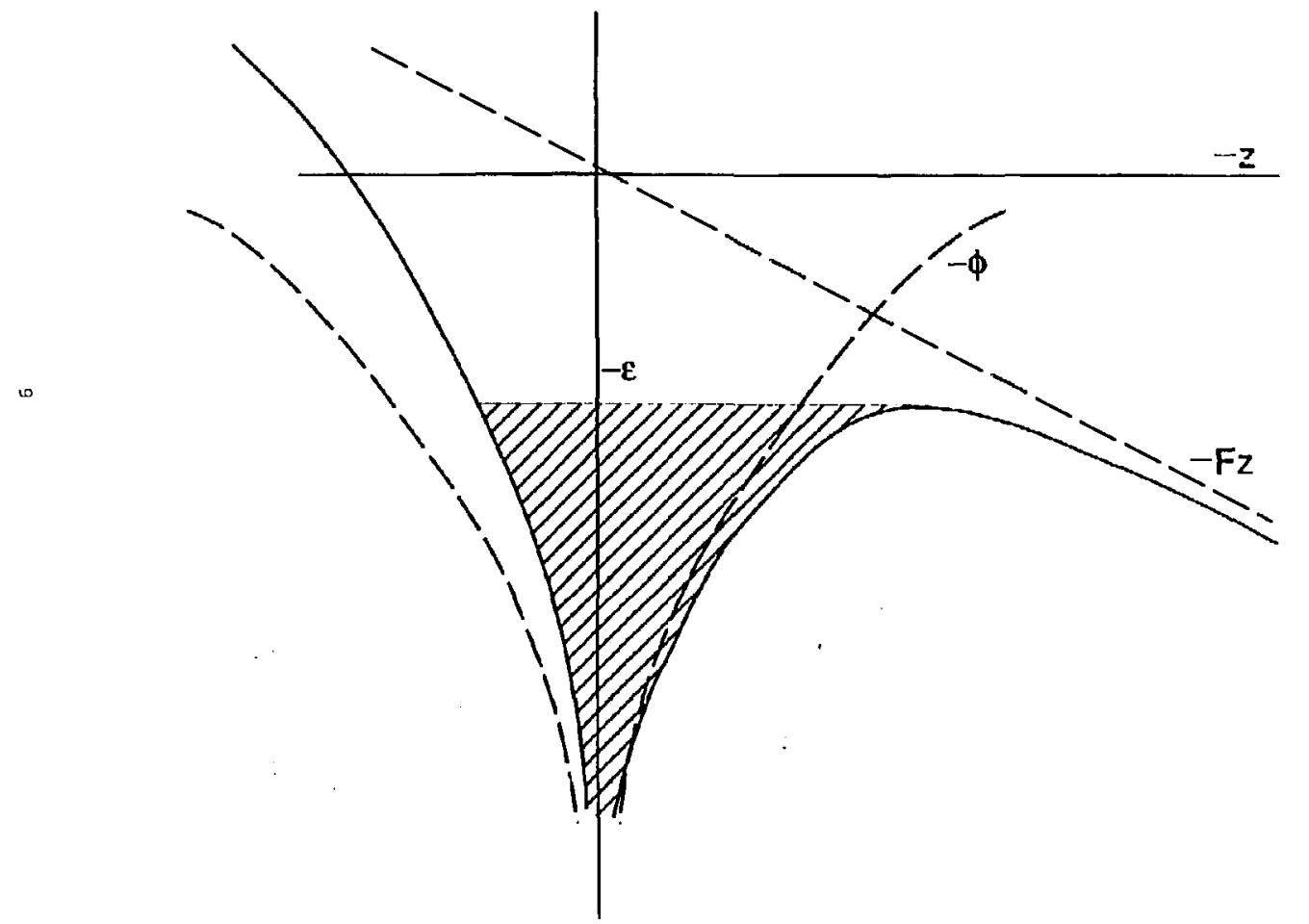

FIG. 1 


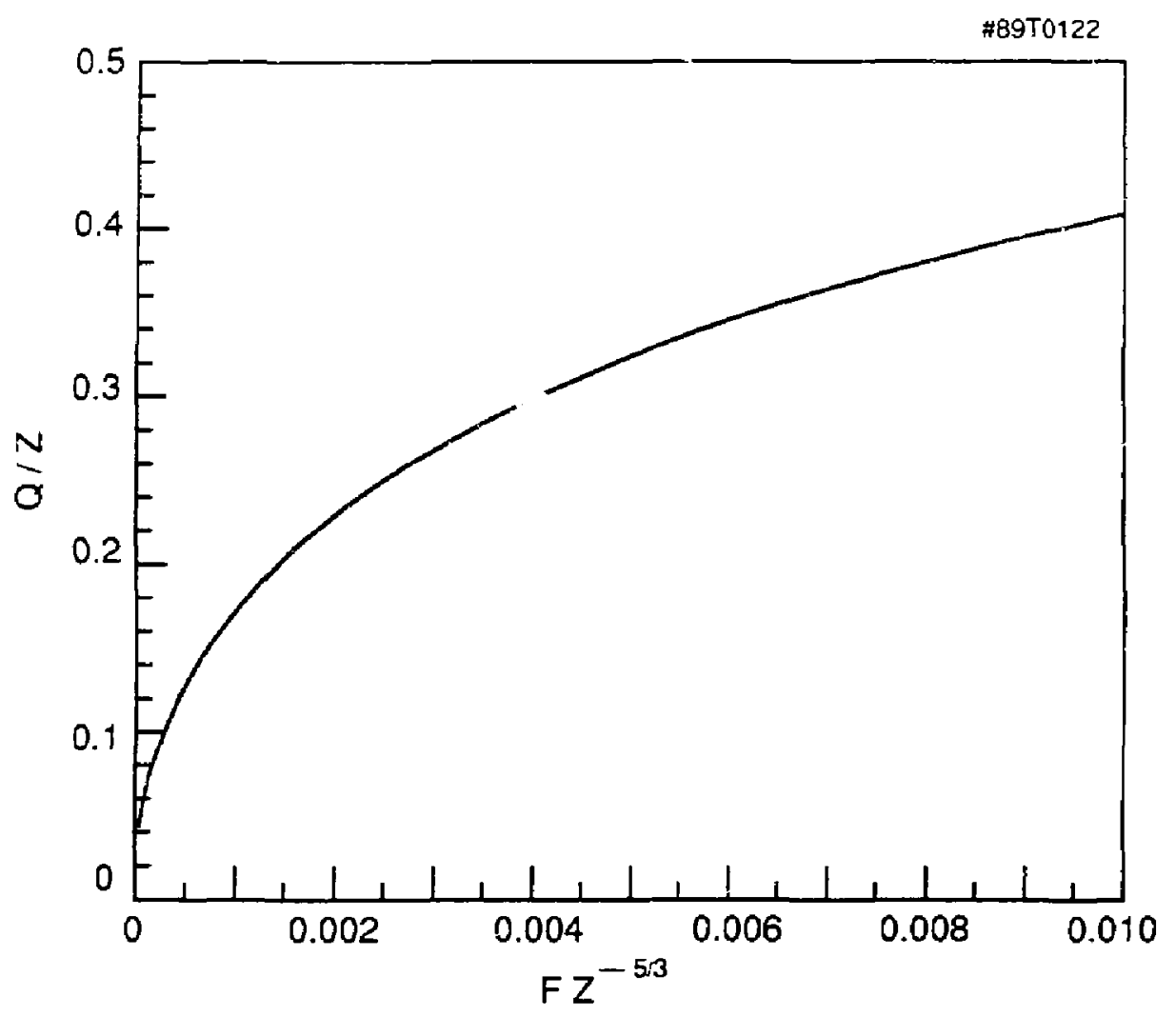

EIG, 2 


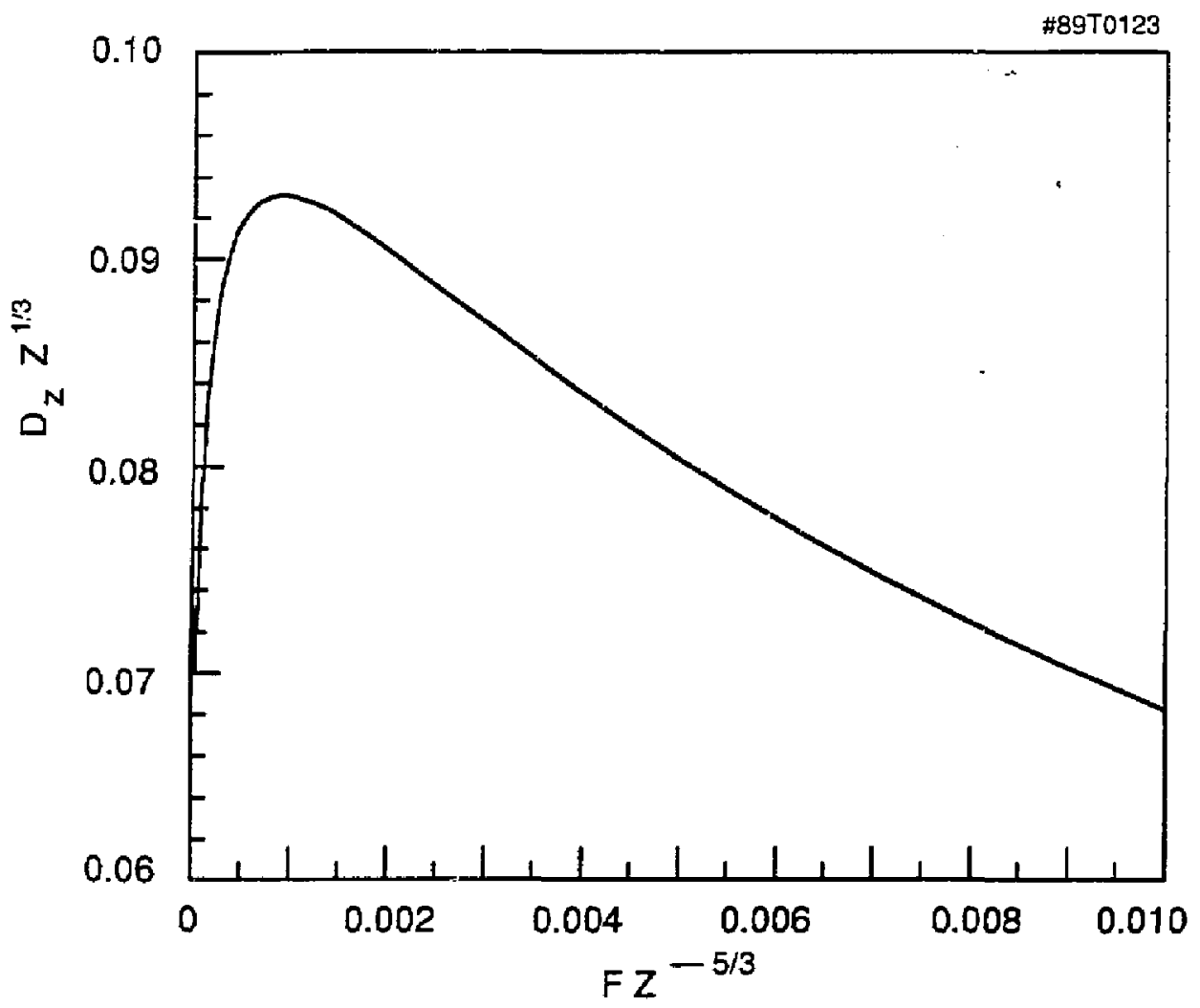

FIG. 3 


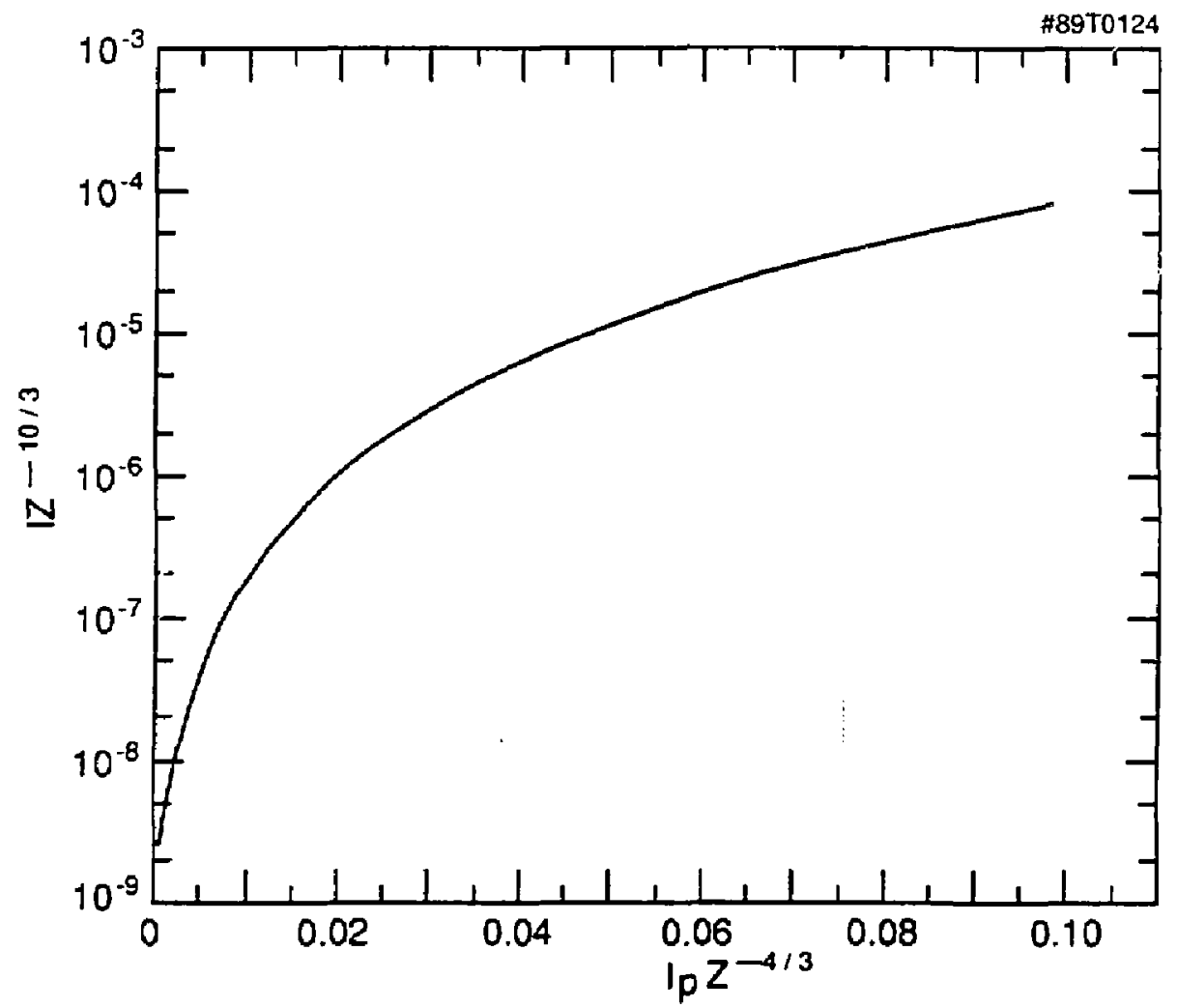

EIG. 4

12 


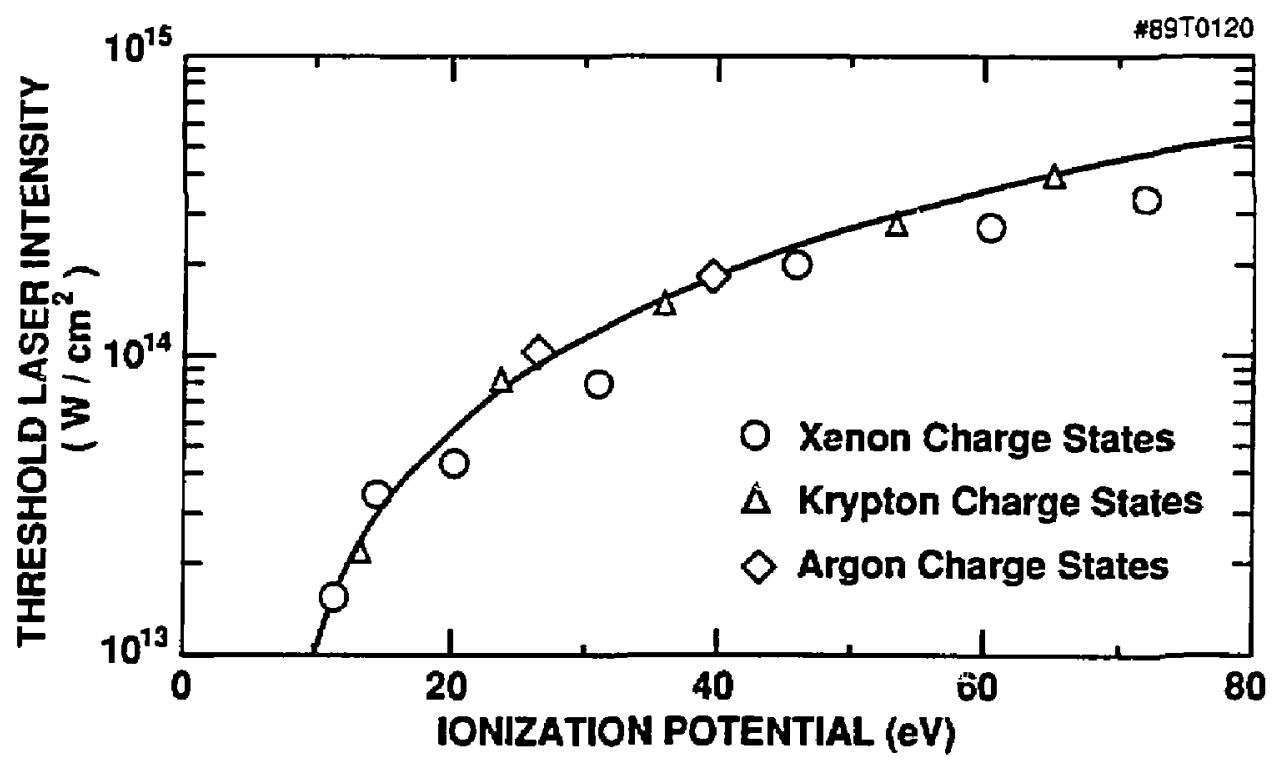

FIG. $5 a$ 


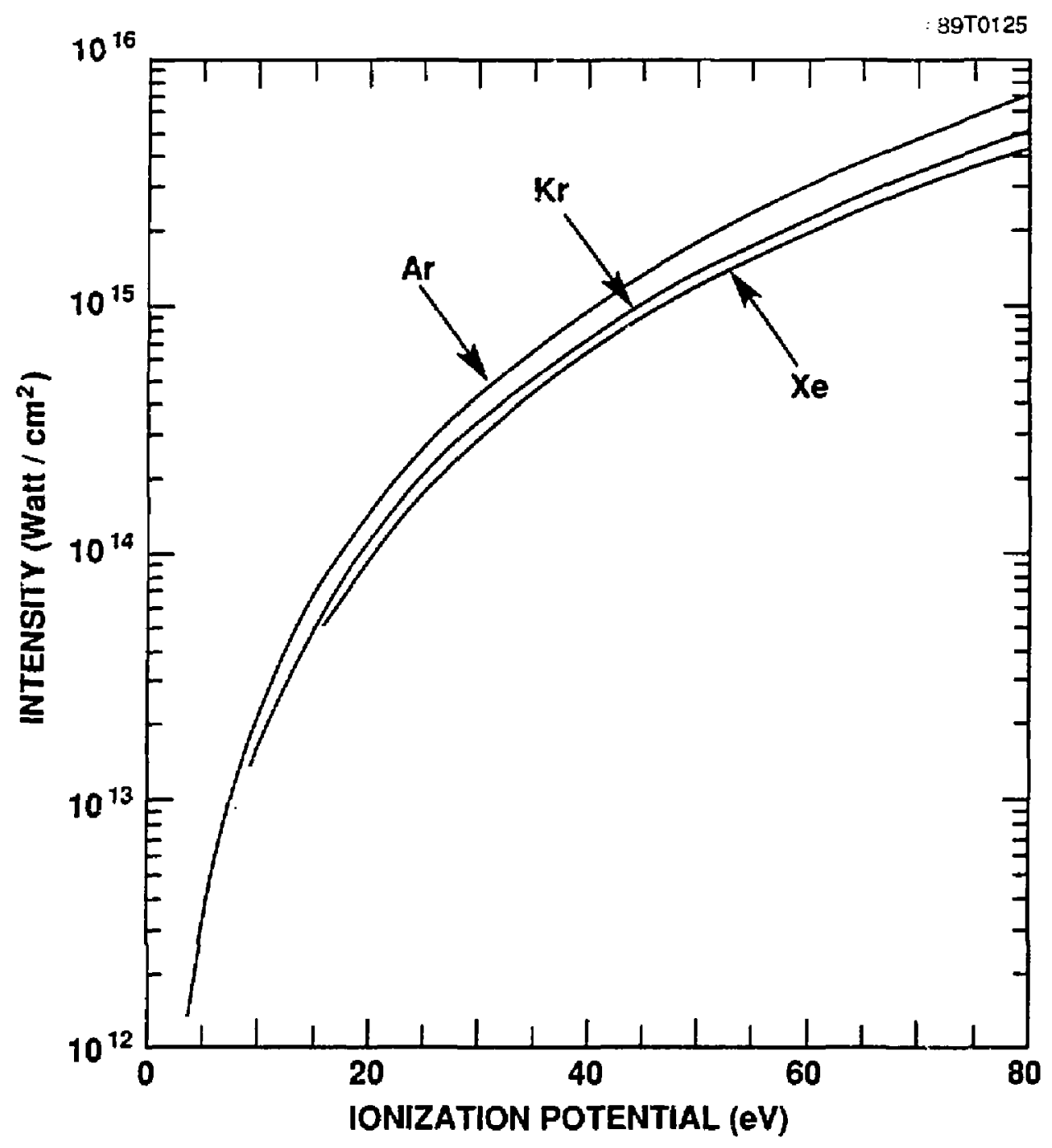

FIG. $5 b$ 